\title{
Qualidade de vida de pacientes pseudofácicos submetidos à cirurgia de catarata com implante de lente intra-ocular acomodativa
}

\author{
Quality oflife of pseudophakic patients with accommodative intraocularlens implant
}

\author{
Filipe de Oliveira ${ }^{1}$ \\ Luci Meire P. Silva ${ }^{2}$ \\ Cristina Muccioli ${ }^{3}$ \\ Eduardo Sone Soriano ${ }^{4}$ \\ Lincoln Leme Freitas ${ }^{5}$ \\ Rubens Belfort Jr. ${ }^{6}$
}

\begin{tabular}{|c|}
\hline RESUMO \\
\hline 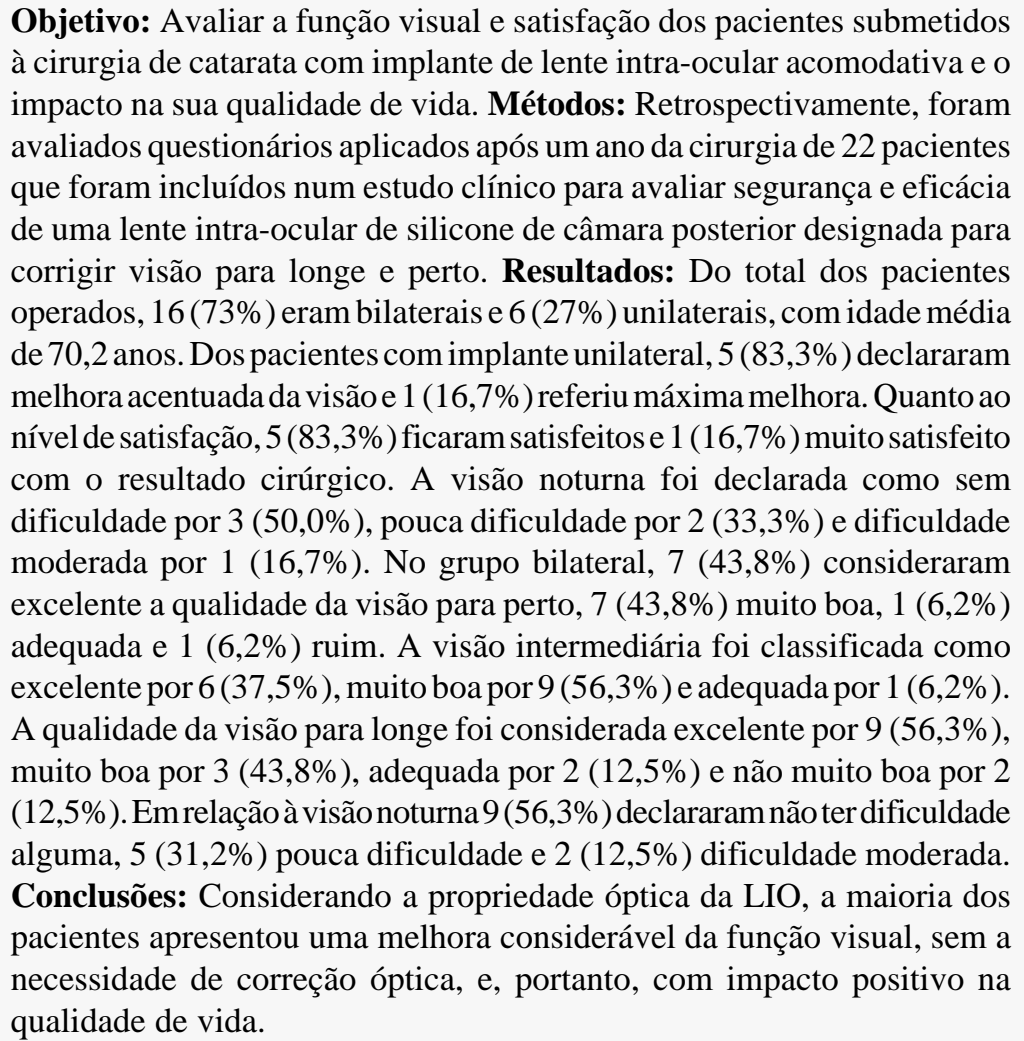 \\
\hline
\end{tabular}

Descritores: Qualidade de vida; Pseudofacia/terapia; Pseudofacia/cirurgia; Extração de catarata; Lentes intra-oculares

\section{INTRODUÇÃO}

A cirurgia de catarata convencional compreende o implante de lentes intra-oculares (LIO) monofocais, que geralmente corrigem a visão para longe. Em 1997, o FDA - Food and Drug Administration aprovou a primeira LIO multifocal, capaz de corrigir a visão para longe e para perto. O surgimento desta tecnologia permitiu melhorar a função visual, satisfação e qualidade de vida dos pacientes ${ }^{(1)}$.

As LIOs convencionais oferecem excelentes resultados visuais à maioria dos pacientes que se submetem à cirurgia de extração de catarata com 
técnicas cirúrgicas modernas. Porém, muitos pacientes necessitam de lente de leitura ou óculos bifocais para a visão de perto, já que a LIO monofocal oferece apenas correção da visão para longe. As LIOs que oferecem correção da visão para longe e para perto, seriam uma alternativa a esses pacientes que procuram correção para a presbiopia pseudofácica.

As LIOs multifocais, apesar de promoverem mais de uma distância focal simultaneamente, produzem uma imagem de qualidade óptica pior do que a produzida por uma LIO monofocal. Esta perda da qualidade de imagem pode afetar a performance visual e, desta forma, promove estudos para se alcançar novas modalidades para a correção da presbiopia pseudofácica $^{(1-5)}$. Estes estudos pressupõem a permanência da função do músculo ciliar a estímulos acomodativos. Existem teorias conflitantes sobre a atrofia do músculo ciliar no decorrer da vida, reforçados por uma série de estudos publicados que demonstram que este músculo perde sua força durante o processo de envelhecimento. Porém, há estudos que demonstram clinicamente a habilidade de um cristalino artificial em restaurar a acomodação em olhos de primatas ${ }^{(4-5)}$.

Em 1989, Cumming iniciou estudos para o desenvolvimento de uma LIO acomodativa. O mecanismo da eficácia desta lente é baseado no conceito de que uma vez que o músculo ciliar encontra-se contraído, há uma redistribuição de sua massa, expandindo-se posteriormente e assim, empurrando a face vítrea perifericamente. Isto aumenta a pressão vítrea, anteriorizando a LIO e contribuindo com seu efeito pseudoacomodativo $^{(5-8)}$.

Packer descreveu o termo função visual como o impacto da visão na qualidade de vida, ou seja, a capacidade de realização de atividades cotidianas estaria diretamente relacionada à funcionalidade visual ${ }^{(9)}$.

O objetivo deste estudo foi avaliar a função visual, bem como a satisfação dos pacientes com implante de LIO acomodativa após extração de catarata, e analisar o impacto na sua qualidade de vida.

\section{MÉTODOS}

Este estudo foi realizado no Departamento de Oftalmologia da UNIFESP/EPM com 22 pacientes com idade entre 53 e 91 anos (média de 70,2 anos) que foram incluídos num estudo clínico investigacional, para avaliar a segurança e eficácia de uma LIO de silicone de câmara posterior designada para corrigir a visão para longe e para perto após a cirurgia de catarata.

Utilizou-se um questionário específico e adaptado para avaliação da qualidade de vida e função visual de pacientes pseudofácicos, e que fora aplicado após um ano da cirurgia. $\mathrm{O}$ questionário englobava questões ligadas à satisfação do paciente na execução de atividades habituais e suas respectivas relações com as visões para longe, intermediária e perto. Além disso, também constavam questões referentes à qualidade da visão noturna e necessidade do uso de óculos (Anexo 1).
Foram analisados os questionários de 16 pacientes $(73 \%)$ com implante bilateral; e dos 6 pacientes (27\%) com implante unilateral, cuja avaliação relacionou-se ao olho com LIO.

Todos os pacientes assinaram um termo de consentimento livre e esclarecido que foi previamente aprovado por Comitê de Ética local, de acordo com a Declaração de Helsinki ${ }^{(10)}$.

\section{RESULTADOS}

Os pacientes com implante monocular responderam questões relacionadas à satisfação com a cirurgia e à qualidade geral da visão (Gráfico 1). Dos pacientes entrevistados, 5 (83,3\%) declararam melhora acentuada da visão, e $1(16,7 \%)$ melhora total. Quanto ao nível de satisfação, $5(83,3 \%)$ ficaram satisfeitos e $1(16,7 \%)$ ficou muito satisfeito com o resultado da cirurgia. Considerando a visão noturna, 3 pacientes $(50,0 \%)$ declararam que não tiveram dificuldade com a visão no período noturno, 2 pacientes $(33,3 \%)$ apresentaram pouca dificuldade $\mathrm{e}$ $1(16,7 \%)$ dificuldade moderada (Gráfico 2). Além disso, 3 pacientes (50\%) do grupo unilateral, referiram ausência de problemas visuais como resultado da cirurgia e $3(50 \%)$ referiram que apresentam alguma dificuldade na visão noturna (Gráfico 2). Todos os pacientes desse grupo se tivessem a oportunidade de escolher, aceitariam colocar a lente desse estudo novamente.

As respostas dos questionários aplicados ao grupo bilateral, cujo teor das questões envolvia a avaliação da qualidade da visão para perto $(40 \mathrm{~cm})$, para distância intermediária $(1 \mathrm{~m}) \mathrm{e}$ para longe (6 m ou mais), são apresentadas no gráfico 3 . A necessidade do uso de óculos após a cirurgia, nesse grupo, foi indicada por 2 pacientes $(12,5 \%)$, que usavam óculos de 10 a $25 \%$ do tempo. Um paciente requereu óculos para perto e o outro para longe (Gráfico 4).

A visão para perto envolvia relação com atividades cotidianas como leitura, costura, entre outras, a visão intermediária englobava a capacidade de ver detalhes de um objeto sobre a mesa, possibilidade de leitura do painel de um veículo, entre outras, e a visão para longe abordava situações como pegar ônibus, caminhar pela rua, ler placas de trânsito, etc. A qualidade da visão noturna também foi considerada de modo que o paciente pudesse relacioná-la a alguma sensação de halo e "glare".

Das entrevistas realizadas com o grupo bilateral, 7 pacientes $(43,8 \%)$ consideraram excelente a qualidade da visão para perto, $7(43,8 \%)$ classificaram como muito boa, $1(6,2 \%)$ considerou adequada e $1(6,2 \%)$ classificou-a como ruim. A visão intermediária foi classificada como excelente por 6 pacientes $(37,5 \%)$, como muito boa por $9(56,3 \%)$ e como adequada por 1 $(6,2 \%)$. A qualidade da visão para longe foi considerada excelente por 9 pacientes $(56,3 \%)$, muito boa por $3(43,8 \%)$, adequada por $2(12,5 \%)$ e não muito boa por 2 pacientes $(12,5 \%)$. Quando questionados sobre a visão noturna, 9 pacientes $(56,3 \%)$ declararam não ter dificuldade alguma, $5(31,2 \%)$ apresentaram pouca dificuldade e $2(12,5 \%)$ relataram dificuldade moderada (Gráfico 2). 


\section{Anexo 1 - Questionários aplicados a cada grupo}

\section{Questionário do Grupo Bilateral}

1) Qual é a freqüência que você usa óculos diariamente?

( ) Eu não uso óculos

( ) Eu uso de $10 \%$ a $25 \%$ do tempo

( ) Eu uso $50 \%$ do tempo

( ) Eu uso de $51 \%$ a $71 \%$ do tempo

( ) Eu uso óculos de $76 \%$ a $100 \%$ do tempo

2) Como você classificaria sua visão sem o uso de óculos?

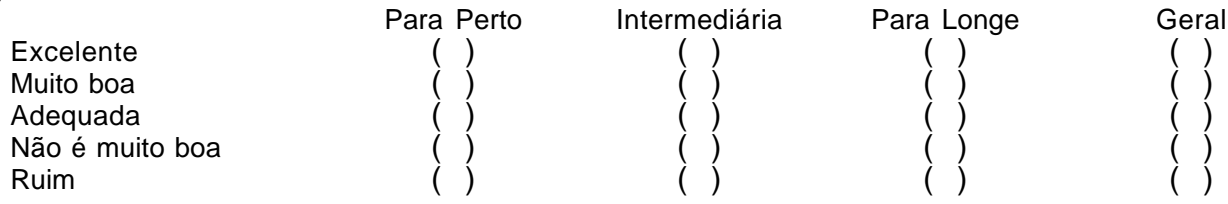

3) Como você classificaria sua visão com uso de óculos? (apenas para os usuários de correção óptica):

\begin{tabular}{|c|c|c|c|}
\hline $\begin{array}{l}\text { Excelente } \\
\text { Muito boa } \\
\text { Adequada } \\
\text { Não é muito boa } \\
\text { Ruim }\end{array}$ & $\begin{array}{l}\text { Para Perto } \\
\text { ( ) } \\
(\text { ) } \\
(\text { ) } \\
(\text { () } \\
(\text { ) }\end{array}$ & $\begin{array}{c}\text { Intermediária } \\
\text { ( ) } \\
(\text { ) } \\
(\text { ) } \\
(\text { () }\end{array}$ & $\begin{array}{c}\text { Para Longe } \\
(\text { ( ) } \\
(\text { ) } \\
(\text { ) } \\
(\text { () }\end{array}$ \\
\hline
\end{tabular}

4) Você usa óculos para enxergar durante a noite? ( ) Sim Não

5) Como você classificaria o nível de dificuldade que você tem para ver durante a noite para as seguintes condições?

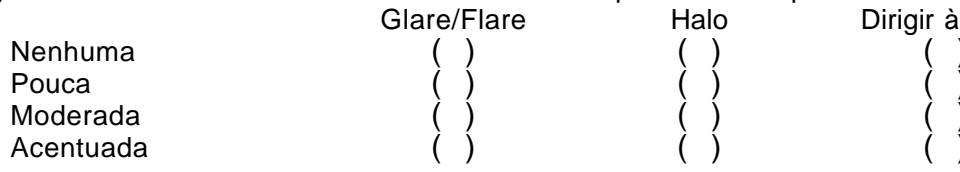

6) Sem o uso de óculos você pode:

\begin{tabular}{|c|c|c|c|}
\hline & Sim & Não & Não aplicável \\
\hline Fazer a maioria das funç & & ( ) & ( ) \\
\hline Ler a maioria das coisas & & & \\
\hline Ir ao shopping & & & \\
\hline Praticar esportes & & & \\
\hline Participar de reuniões so & & & \\
\hline Dirigir / Ler placas de trâr & & & \\
\hline Ler jornal & & & \\
\hline Costurar & & & \\
\hline Usar computador & & & \\
\hline Caminhar / Tomar ônibus & & & \\
\hline Fazer compras & & & \\
\hline Assistir TV & & & ) \\
\hline
\end{tabular}

\section{Questionário do Grupo Unilateral}

1) Atualmente você apresenta problemas visuais como resultado de sua cirurgia? $\quad$ ？ ） Sim ( ) Não Se sim, indique a extensão desse problema de acordo com as seguintes condições:

Sensibilidade à luz

Glare

Halos

Visão noturna (dirigir/caminhar)

Flutuação da visão
Moderado

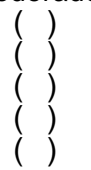

(

2) Atualmente como você classificaria a melhora da sua visão se comparada com a visão antes da sua cirurgia?
( ) Máxima
( ) Acentuada
( ) Moderada
( ) Leve
( ) Ausente

3) Se você tivesse que escolher, você aceitaria colocar esta lente novamente?

( ) Sim

( ) Não

4) Como você se sente em relação à satisfação com os resultados de sua cirurgia de catarata?
( ) Muito satisfeito
( ) Moderadamente satisfeito
( ) Neutro
( ) Insatisfeito
( ) Muito insatisfeito 


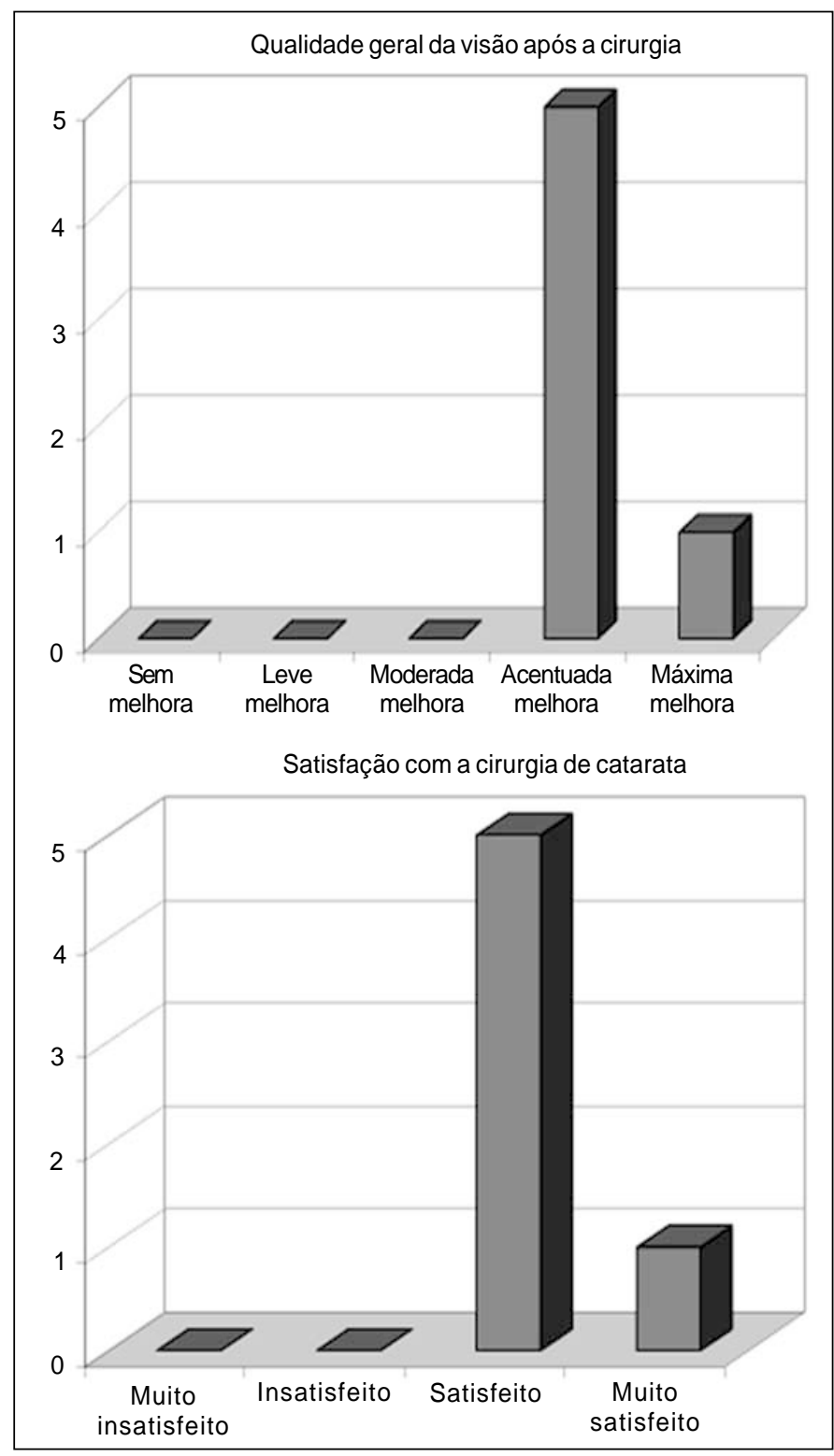

Gráfico 1 - Qualidade geral da visão e satisfação dos pacientes após a cirurgia de catarata (Implantes unilaterais [N=6])

A classificação da qualidade visual geral sem o uso de correção óptica no grupo bilateral, foi considerada excelente por 6 pacientes (38\%), muito boa por $8(49 \%)$ e adequada por 2 (13\%) (Gráfico 5), sendo que os mesmos responderam que realizam positivamente a maioria das tarefas e funções do cotidiano (Anexo 1- questão 6).

\section{DISCUSSÃO}

Considerando as respostas de cada paciente do grupo bilateral, verificamos que a LIO acomodativa utilizada neste estudo proporcionou um impacto positivo na qualidade geral de vida destes pacientes. Com a independência do uso de óculos, os

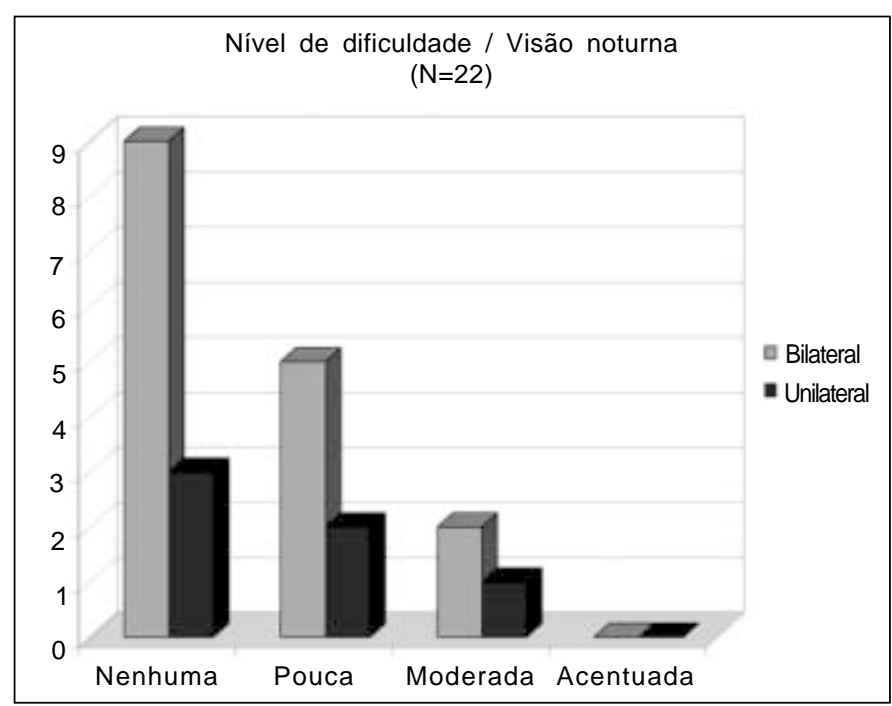

Gráfico 2 - Qualidade da visão noturna

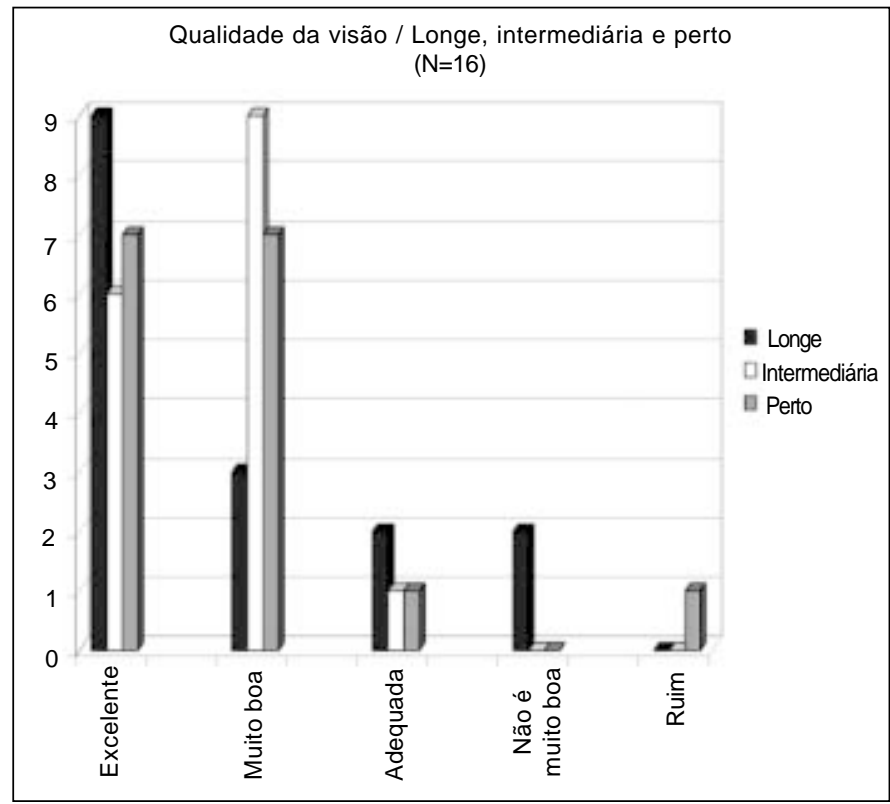

Gráfico 3 - Qualidade geral da visão / Grupo bilateral

mesmos retomaram atividades que eram impossíveis antes da extração da catarata, como, por exemplo, dirigir, caminhar, ler jornais, pegar ônibus, ver preços no supermercado, entre outras. O perfil destas respostas condiz com alguns estudos precisos realizados com implante de LIO multifocal ${ }^{(1,11-12)}$.

No grupo de pacientes com implante unilateral, o olho com implante apresentava uma acuidade visual consideravelmente melhor se comparada à do olho contralateral. Convém ressaltar que a visão binocular normal estaria restabelecida com o implante bilateral.

A qualidade da visão noturna para ambos os grupos, representou um importante fator de eficácia desta LIO, pois possibili- 


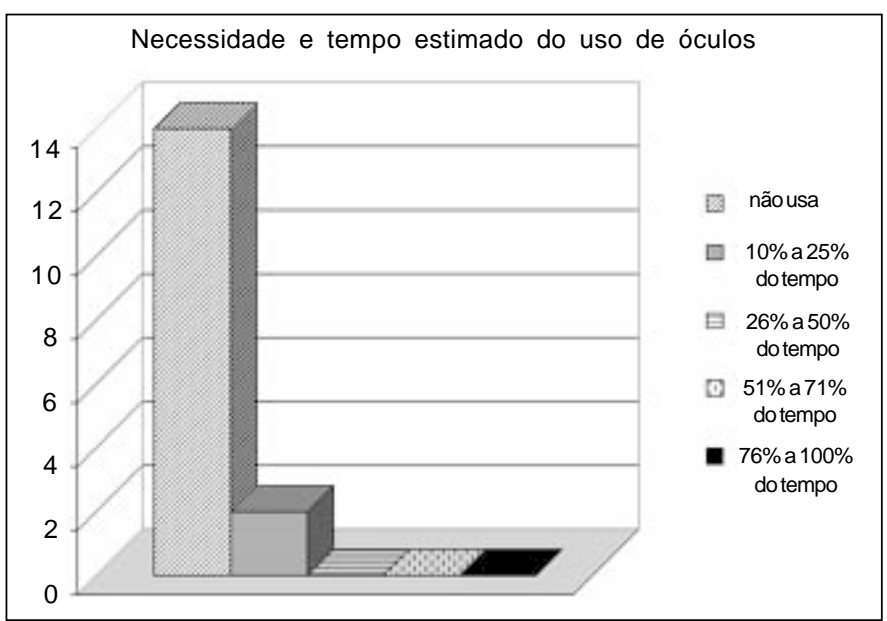

Gráfico 4 - Necessidade de correção óptica

tou aos pacientes executarem bem atividades habituais como dirigir ou caminhar à noite, sem influência de fatores como halos ou "glare" que pudessem comprometer estas atividades neste período do dia. Diferente do encontrado em alguns estudos com implante de LIO multifocal, onde nos questionários de avaliação da função visual e qualidade de vida, os pacientes relataram incômodo relacionado a halo e "glare"(1,11).

\section{CONCLUSÃO}

De acordo com análise dos questionários, concluímos que após um ano da cirurgia, a LIO acomodativa respeitou bem sua propriedade óptica, proporcionando melhora da acuidade visual para longe, para meia-distância (intermediária) e para perto. A maioria dos pacientes $(90,9 \%)$ envolvidos neste estudo apresentaram uma melhora considerável da função visual sem a dependência de uso de correção óptica.

Com o presente estudo, confirmamos o impacto positivo do uso de lentes intra-oculares com efeito pseudoacomodativo, na melhora da qualidade de vida dos pacientes portadores de catarata senil.

\section{ABSTRACT}

Purpose: To evaluate the visual function and satisfaction of patients who underwent cataract surgery and were implanted with an accommodative intraocular lens and the impact in their quality of life. Methods: Retrospectively, questionnaires applied one year after cataract surgery of 22 patients who were included in a clinical trial to evaluate the safety and effectiveness of a silicone multipiece intraocular lens designed to provide distance and near vision were analyzed. Results: Twenty-two patients with mean age of 70.2 years were enrolled in the study. Sixteen (73\%) patients received bilateral and $6(27 \%)$ unilateral implants of the unilateral implant group,

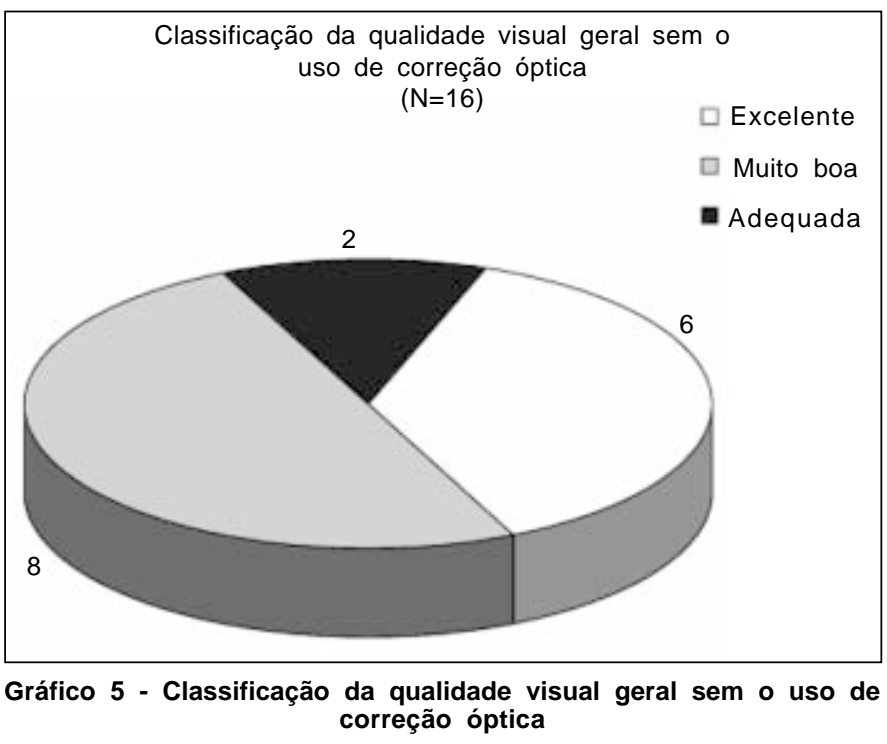

$5(83.3 \%)$ patients declared marked improvement of vision, $1(16.7 \%)$ referred maximum improvement. Regarding satisfaction, $5(83.3 \%)$ declared to be satisfied and $1(16.7 \%)$ was very satisfied with the results of the surgery in general. Night vision was declared as without difficulty by 3 patients $(50.0 \%)$, mild difficulty by $2(33.3 \%)$ and moderate difficulty by $1(16.7 \%)$. In the bilateral group, $7(43.8 \%)$ considered near vision to be of excellent quality, 7 (43.8\%) very good, $1(6.2 \%)$ adequate and $1(6.2 \%)$ poor. The quality of intermediate vision was classified as excellent by $6(37.5 \%)$ patients, very good by $9(56.3 \%)$ and adequate by $1(6.2 \%)$. The quality of distance vision was considered excellent by $9(56.3 \%)$ patients, very good by $3(43.8 \%)$, adequate by $2(12.5 \%)$ and not very good by $2(12.5 \%)$. Regarding night vision, $9(56.3 \%)$ patients declared no difficulty, 5 (31.2\%) mild difficulty and $2(12.5 \%)$ moderate difficulty. Conclusion: Considering IOL optical property, most patients presented important improvement of visual function, without need to wear spectacles and with positive impact on their quality of life.

Keywords: Quality of life; Pseudophakia/therapy; Pseudophakia/surgery; Cataract extraction; Lenses, intraocular

\section{REFERÊNCIAS}

1. Javitt JC, Steinert RF. Cataract extraction with multifocal intraocular lens implantation: a multinational clinical trial evaluating clinical, functional and quality-of-life outcomes. Ophthalmology 2000;107:2040-8.

2. Nishi O, Nakai Y, Yamada Y, Mizumoto Y. Amplitudes of accommodation of primate lenses refilled with two types of inflatable endocapsular ballons. Arch Ophthalmol 1993;111:1677-84.

3. Haefliger E, Parel JM, Fantes F, Norton EW, Anderson DR, Forster RK, Hernandez E, Feuer WJ. Accommodation of an endocapsular silicone lens (Phaco-Ersatz) in nonhuman primate. Opthalmology 1987;94:471-7.

4. Haefliger E, Parel JM. Accommodation of an endocapsular silicone lens (PhacoErsatz) in the aging rhesus monkey. J Refract Corneal Surg 1994;10:550-5. 
5. Bito LZ, De Rosseau CJ, Kaufman PL, Bito JW. Age-dependent loss of accommodative amplitude in rhesus monkeys: an animal model for presbyopia. Invest Ophthalmol Vis Sci 1982;23:23-31.

6. Fisher RF. The mechanics of accommodation in relation to presbyopia. Eye 1988;2:646-9.

7. Humber C. Planned myopic astigmatism as a substitute for accommodation in pseudophakia. Am Intraocular Implant Soc J 1981;7:244-49.

8. Cumming JS. Lente intra-ocular com capacidade de acomodação. Oftalmologia em Foco 2000/2001;70:11.

9. Packer M, Fine IH, Hoffman RS. Functional vision, contrast sensitivity, and optical aberrations. Int Ophthalmol Clin 2003;43:1-3.
10. Declaration of Helsinki: recomendations guiding Medical doctors in biomedical research involving human subjects. 41 st World Medical Assembly. Hong Kong: World Medical Association; 1989.

11. Javitt JC, Wang F, Trentacost DJ, Rowe M, Tarantino N. Outcomes of cataract extraction with multifocal intraocular lens implantation: functional status and quality of life. Ophthalmology 1997;104:589-99.

12. Steinert RF, Aker BL, Trentacost DJ, Smith PJ, Tarantino N. A prospective comparative study of the AMO ARRAY zonal-progressive multifocal silicone intraocular lens and a monofocal intraocular lens. Ophthalmology 1999;106: 1243-55.

\section{SIMPÓSIO DA SOCIEDADE BRASILEIRA DE GLAUCOMA}

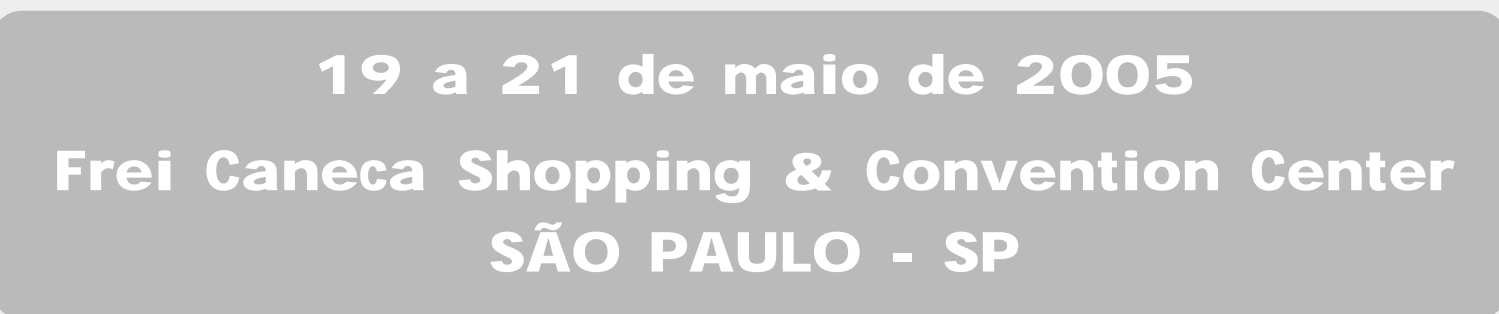

IN FO RMAÇÕ ES: JDE Comunicação e Eventos

Tels.: (11) 5082-3030 / 5084-5284

Fax: (11) 5574-8261

e-mail: jdecomev@uol.com.br 